\title{
Magnetic two-dimensional electron liquid at the surface of Heusler semiconductors
}

\author{
S. Keshavarz, ${ }^{1}$ I. Di Marco, ${ }^{1,2,3}$ D. Thonig, ${ }^{1,4}$ L. Chioncel,${ }^{5,6}$ O. Eriksson,,${ }^{1,4}$ and Y. O. Kvashnin ${ }^{1}$ \\ ${ }^{1}$ Department of Physics and Astronomy, Division of Materials Theory, Uppsala University, Box 516, SE-751 20 Uppsala, Sweden \\ ${ }^{2}$ Asia Pacific Center for Theoretical Physics, Pohang, Gyeongbuk 790-784, Korea \\ ${ }^{3}$ Department of Physics, POSTECH, Pohang, Gyeongbuk 790-784, South Korea \\ ${ }^{4}$ School of Science and Technology, Örebro University, SE-701 82 Örebro, Sweden \\ ${ }^{5}$ Theoretical Physics III, Center for Electronic Correlations and Magnetism, Institute of Physics, \\ University of Augsburg, D-86135 Augsburg, Germany \\ ${ }^{6}$ Augsburg Center for Innovative Technologies, University of Augsburg, D-86135 Augsburg, Germany
}

(Received 24 May 2019; accepted 19 February 2020; published 28 February 2020)

\begin{abstract}
Conducting and magnetic properties of a material often change in some confined geometries. However, a situation where a nonmagnetic semiconductor becomes both metallic and magnetic at the surface is quite rare, or maybe even unique. In this Rapid Communication, we employ first-principles electronic structure theory to predict that such a peculiar magnetic state emerges in a family of quaternary Heusler compounds. We investigate magnetic and electronic properties of CoCrTiP, FeMnTiP, and CoMnVAl. For the latter material, we also analyze the structural stability of various surface terminations. For the ideal CoMn termination we calculate the magnetic exchange interactions and use them for parametrizing an effective spin Hamiltonian. According to our results, magnetism in this material should persist at temperatures at least as high as $160 \mathrm{~K}$.
\end{abstract}

DOI: 10.1103/PhysRevMaterials.4.021401

Introduction. In several classes of materials, surfaces are known to exhibit properties distinctly different from those of the bulk. At the quantum mechanical level, surface electronic states were first characterized almost a century ago, in the pioneering works of Tamm [1] and Shockley [2]. A direct probe of surface states became possible only much later, with the improvements in experimental techniques, as, e.g., in angle-resolved photoelectron spectroscopy (ARPES) [3-6]. The arising of surface physics led to the observation of several key phenomena. Materials characterized by a band gap in their bulk electronic structure can possess a metallic surface hosting a two-dimensional electron gas (2DEG), as, e.g., in $\mathrm{SrTiO}_{3}$ [7], or the much celebrated topological edge modes [8]. Differences between surface and bulk electronic structures are also the foundation of interface-induced effects, as those observed when combining $\mathrm{LaAlO}_{3}$ and $\mathrm{SrTiO}_{3}$. The interface of these two insulators does not only host a peculiar 2DEG [9-11], but may also harbor a quasi-2D magnetic order driven by oxygen defects $[12,13]$. These studies associate the interface magnetism with off-stoichiometry modeled by artificially ordered supercells, sometimes limited to high defect concentrations $[14,15]$.

Confined geometries have always been of primary importance for Heusler and half-Heusler compounds [16]. For instance, understanding interfaces is crucial for building

Published by the American Physical Society under the terms of the Creative Commons Attribution 4.0 International license. Further distribution of this work must maintain attribution to the author(s) and the published article's title, journal citation, and DOI. Funded by Bibsam.
Heusler-based devices for giant and tunneling magnetoresistances [16-18]. Interfaces have not only a practical importance, but may also host noncollinear spin ordering $[19,20]$ resulting in complex magnetic structures. Recently, materials such as LaPtBi, LuPtSb, ScPtBi, YPdBi, and ThPtPb were shown to represent a new class of three-dimensional topological insulators [21].

Due to the high tunability and versatility of Heusler compounds, new phenomena are expected to emerge when more and more materials are suggested and synthesized. Precisely, in this Rapid Communication, we predict that a relatively unexplored (but already existing [22]) family of quaternary Heusler compounds hosts a rare, if not unique, phenomenon, where a semiconducting and nonmagnetic bulk coexists with a metallic surface with robust magnetism. By means of firstprinciples electronic structure calculations, we show this peculiar state to emerge in CoMnVAl, CoCrTiP, and FeMnTiP. Using calculated exchange interactions, we illustrate that the magnetic long-range order at the surface is stable up to sizable temperatures, which is a fundamental prerequisite for experimental verification. We discuss the results both in terms of fundamental understanding of this class of electronic structures, as well as in terms of technological aspects, that may emerge when spin degrees of freedom enter semiconductorbased electronic devices [23-27].

To the best of our knowledge, the coexistence of a semiconducting and nonmagnetic bulk with a metallic and magnetic surface still needs to be observed in experiment. Here. we identify a promising prototype of such materials, which are also characterized by a spin-polarized 2D electron liquid emerging at the surface. It is important to stress that differentiating liquid and gas is not only a naming convention. Localized $3 d$ orbitals, which give rise to the surface metallicity 
and magnetism here, are likely to harbor strong electronic correlations, which may lead to a plethora of exotic effects. Half-metallic (HM) Heusler compounds are known to host a wide array of such features. For example, genuine many-body states, named nonquasiparticle states, may appear in HM ferromagnets, leading to a reduction of spin polarization [28-31]. Another example is given by the mass enhancement and non-Fermi-liquid behavior observed in $\mathrm{Fe}_{2} \mathrm{VAl}$ and related materials [16]. These effects, as well as others, are expected to emerge in the reported systems, due to the considerable localization and reduced dimensionality.

Theory and methods. Density functional theory (DFT) calculations were carried out using the full-potential linear muffin-tin orbital (FP-LMTO) code RSPT [32,33]. We used the generalized gradient approximation (GGA), in the formulation of Perdew, Burke, and Enzerhof [34]. The crystal structure of the considered class of Heusler semiconductors is described by a chemical formula $X X^{\prime} Y Z$, where $X, X^{\prime}$, and $Y$ are transition metals and $Z$ is an $s p$ element [35]. The surface was modeled in a supercell geometry consisting of four unit cells stacked along the [001] direction. This means that eight (or nine) $X X^{\prime}$ layers mediated by eight alternating $Y Z$ layers were considered [36]. A vacuum of $25 \AA$ thickness has been added to ensure no interaction between the surfaces. We have calculated equilibrium lattice constants for the bulk and then relaxed all atomic positions for the slab geometries using VASP $[37,38]$. The details of these calculations as well as the relevant references [39-46] can be found in the Supplemental Material (SM) [47].

In addition to standard GGA, we also performed calculations where the effects of strong electron-electron repulsion were included explicitly, at the level of a mean-field DFT $+U$ approach $[48,49]$. For simplicity, we adopted the same values of the Coulomb interaction parameters for the $d$ states of all $X$ and $X^{\prime}$ elements, namely, $U=2 \mathrm{eV}$ and $J=0.8 \mathrm{eV}$. These values are situated in between the partly and fully screened estimates [50], obtained for similar compounds using the constraint random phase approximation [51].

The thermal stability of the predicted magnetic long-range order at the surface was investigated by means of an effective Heisenberg model. The latter is described by a Hamiltonian $\hat{H}=-\sum_{i \neq j} J_{i j} \vec{e}_{i} \cdot \vec{e}_{j}$, where $J_{i j}$ is the exchange interaction between the spins located at the site $i$ and $j$, and $\vec{e}$ is a unit vector along the spin direction at the corresponding site. The $J_{i j}$ 's were extracted from a GGA $(+U)$ calculation by means of the magnetic force theorem [52-54]. Based on these values of the $J_{i j}$ 's, we calculated the ordering temperature $\left(T_{c}\right)$ by means of a classical Monte Carlo (MC) simulation, as implemented in the UPPASD code $[55,56]$. The adiabatic magnon spectra (AMS) were calculated for a considered magnetic ground state from the $J_{i j}$ 's.

Results and discussion. We first analyze the bulk properties of three Heusler semiconductors: CoMnVAl, CoCrTiP, and FeMnTiP. According to the Slater-Pauling rule [57,58], Heusler compounds with 24 valence electrons are expected to have zero net moment. They can either represent compensated ferrimagnetic half metals or semiconductors with antiferromagnetic or nonmagnetic orders $[59,60]$. Our results indicate that the selected materials belong to the latter category, in agreement with previous literature [61]. The calculated band
TABLE I. Calculated site-projected spin moments $\left(\mu_{B}\right)$ for the considered Heusler compounds of $X X^{\prime} Y Z$ family. The GGA $+U-$ derived results are shown in parentheses. The indices refer to the layer numbers from the surface: surface (1), subsurface (2), and subsubsurface (3).

\begin{tabular}{lccc}
\hline \hline & CoMnVAl & FeMnTiP & CoCrTiP \\
\hline$X_{1}$ & $0.86(1.28)$ & $1.82(2.44)$ & $0.40(0.56)$ \\
$X_{1}^{\prime}$ & $3.71(3.96)$ & $3.75(3.98)$ & $3.50(3.66)$ \\
$Y_{2}$ & $-0.31(-0.76)$ & $-0.28(-0.41)$ & $-0.09(-0.15)$ \\
$X_{3}$ & $0.08(0.09)$ & $0.08(0.07)$ & $0.20(0.25)$ \\
$X_{3}^{\prime}$ & $-0.07(0.01)$ & $-0.08(-0.02)$ & $0.04(0.08)$ \\
\hline \hline
\end{tabular}

gaps in GGA $(\mathrm{GGA}+U)$ are 0 (0.22) for CoMnVAl, $0.2(0.37)$ for CoCrTiP. and 0.44 (0.81) for FeMnTiP, where all values are given in $\mathrm{eV}$. The previous values show that GGA predicts CoMnVAl to be metallic, which is remedied by the inclusion of the $U$ term. The situation is reminiscent of $\mathrm{Co}_{2} \mathrm{FeSi}$, where on-site correlations are also needed to obtain the true HM solution $[62,63]$. CoCrTiP and FeMnTiP are already semiconducting in standard GGA, but the band gaps are further enhanced when $U$ is added.

Next, we focus on the finite-size slabs, starting with the analysis of their spectral properties. For the moment, we limit our analysis to the $X X^{\prime}$ termination, but other terminations will be discussed below. The projected density of states (DOS) for the atoms closest to the surface is reported in Fig. 1, for all three considered systems. The DOS of the deeper-lying layers quickly converges to that of bulk materials (see SM [47] for details). The innermost layers of the 16-layer slab are indistinguishable from the bulk, with spin-degenerate bands, as was also reported in Ref. [20]. The fundamental feature of Fig. 1 is that all systems are characterized by magnetic and metallic DOS at the surface and subsurface layers. These localized surface states which originate from the $3 d$ orbitals of the transition-metal atoms are the ones responsible for the emergence of magnetism.

The projected magnetic moments in the surface and subsurface layers are reported in Table I. Transition-metal atoms at the surface exhibit pronounced magnetic moments, which are aligned ferromagnetically with each other. The Y atoms (V or Ti) belonging to the subsurface layer show weak magnetic moments, which are antiparallel to the large moments at the surface, thus resulting in a ferrimagnetic order. The third layer below the surface is already nearly spin degenerate.

We note that the inclusion of the strong electron-electron repulsion via the local $U$ term does not change the qualitative picture obtained in plain GGA. In the projected DOS of Fig. 1, the localization induced by the $U$ term leads to slightly narrower bands and larger spin splittings. Accordingly, the magnetic moments reported in Table I are moderately enhanced in the DFT $+U$ approach, when compared to the values obtained in GGA.

In general, the surface of a nonmagnetic material can become magnetic because of a reduced number of neighboring atoms, which leads to a narrower bandwidth and the fulfillment of the Stoner criterion. In fact, calculations for the nonmagnetic phase reveal that the surface DOS shows high 

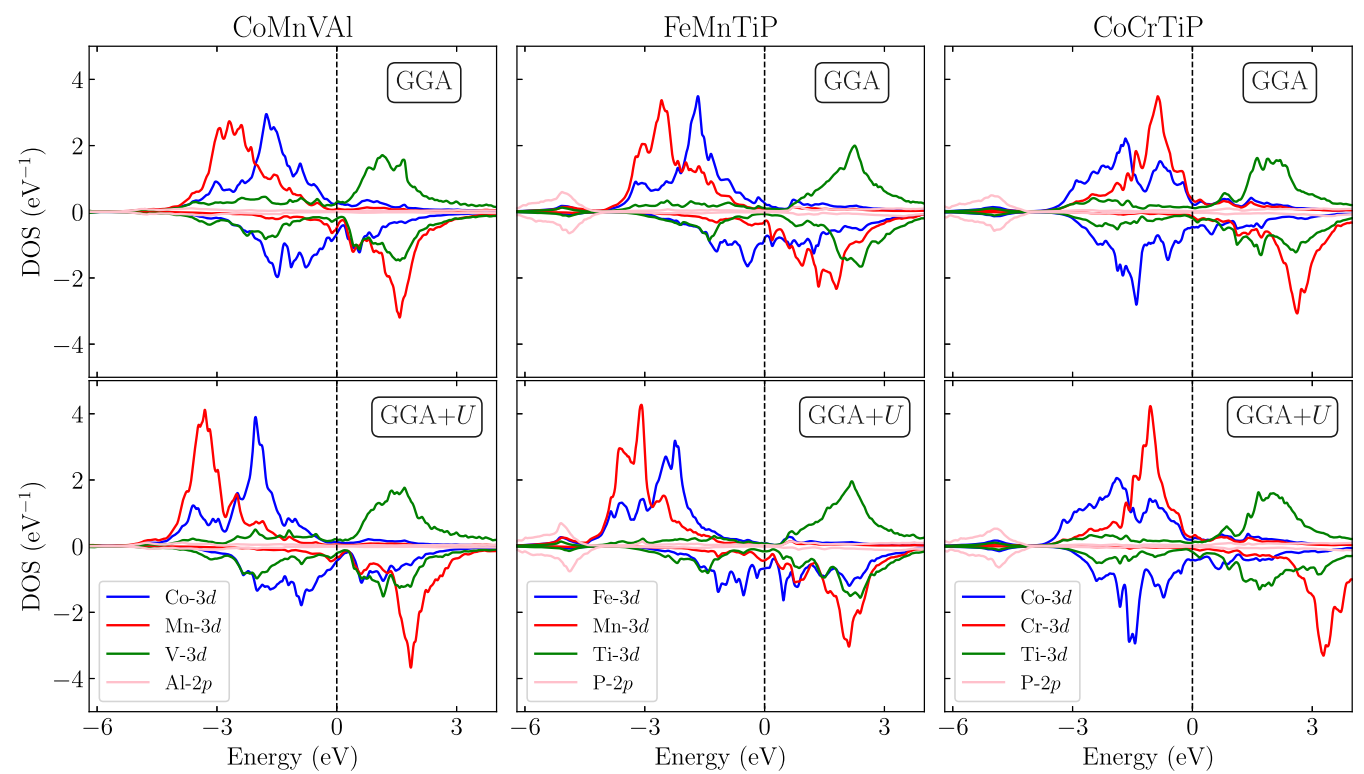

FIG. 1. Projected density of states of the surface atoms (blue and red lines) and the subsurface atoms (green and pink lines) obtained with GGA (top panel) and GGA $+U$ (bottom panel). The Fermi level is indicated by the dashed line. Atomic relaxations were performed in all considered cases.

peaks at the Fermi level, due to the aforementioned surface states. Thus, the Stoner criterion is fulfilled, and the spin degeneracy is removed (see SM [47] for more details). However, this class of materials exhibits yet another peculiarity, which is the Slater-Pauling rule. The latter dictates the lack of magnetism in the bulk, as a consequence of having 24 valence electrons in the unit cell. However, this constraint no longer holds at the surface, due to the dangling bonds caused by the reduced dimensionality. Therefore, it is almost natural for magnetism to arise. An analogous effect was previously reported for Heusler ferromagnets, whose surfaces were shown to exhibit a strongly modified electronic structure, lacking the half metallicity of the bulk [64].

For a more realistic description of these systems, one has to investigate possible surface reconstructions. An extended study of the $\mathrm{Co}_{2} \mathrm{MnSi}(001)$ surface was conducted in Ref. [41]. It was shown that the resulting phase diagram is quite rich and that off-stoichiometric terminations can be stabilized under certain conditions. Using the same approach [40], we have studied 18 various terminations of the CoMn$\operatorname{VAl}(001)$ surface [47]. It is worth noting that all terminations which we considered exhibit both magnetism and metallicity at the surface. The most preferable terminations are identified to be AlMn, VAl, and CoMn, and the differences between their surface energies are quite small [47]. Our analysis based on the calculated phonon dispersions suggests that CoMn and AlMn terminations are dynamically stable, whereas the VAl termination is likely not (see Fig. S4 in SM [47]). Thus, we can conclude that the CoMn termination is at least a metastable solution and should be experimentally realizable.

The previous analysis illustrates that magnetism and metallicity are basically confined to the surface and subsurface layers. Both features are due to localized surface states of $3 d$ character, which are expected to exhibit a substantial electronelectron interaction. Therefore, we refer to the observed situation as the formation of a (quasi-)2D electron liquid, to be distinguished from the 2DEG observed at certain interfaces, as discussed in the Introduction.

To get deeper insight into the origin of the magnetic order at the surface, we have calculated the interatomic exchange parameters $J_{i j}$ 's for CoMnVAl. The structure of the considered slab as well as the calculated $J_{i j}$ 's are reported in Fig. 2. The important couplings are those involving $\mathrm{Co}, \mathrm{Mn}$, and $\mathrm{V}$ atoms that are closest to the surface [65]. The dominant interactions correspond to the nearest-neighbor (NN) Co-Mn, $\mathrm{Co}-\mathrm{V}$, and $\mathrm{Mn}-\mathrm{V}$ bonds. Their signs are perfectly consistent with the ground-state magnetic order, where surface and subsurface spins are antiparallel to each other. Taking the $U$ term into account does not change this picture, but results in an enhancement of all couplings, except the one between Co and $\mathrm{Mn}$. The largest variation is observed for the interactions involving $\mathrm{V}$ atoms, and is partly induced by the large increase of the $\mathrm{V}$ moment (see Table I). The NN couplings between the atoms of the same element (e.g., Mn-Mn) are strongly bond dependent. This is a manifestation of the $C_{2 v}$ symmetry of the slab, which makes the $x$ and $y$ direction nonequivalent. In practice, this implies that the corresponding bonds have different environments and two of them have $\mathrm{Al}$ atoms positioned below and for the other two there are $\mathrm{V}$ atoms. These vastly different exchange paths affect the sign and the magnitude of the $J_{i j}$. The antiferromagnetic NN Co-Co, Mn-Mn. and V-V interactions are, in fact, frustrated.

Electronic structure calculations demonstrate the formation of a spontaneous surface magnetization at zero temperature. To assess the stability of the magnetic state at finite temperature, we performed MC simulations for the parametrized Heisenberg model. For CoMnVAl, the $T_{c}$ is predicted to be in the range between 160 and $180 \mathrm{~K}$, as obtained in GGA and GGA $+U$, respectively. Such a similarity between these results indicates a high confidence in the predicted values. Further information can be obtained through the AMS. The spectrum of CoMnVAl, reported in Fig. 3, shows that the 

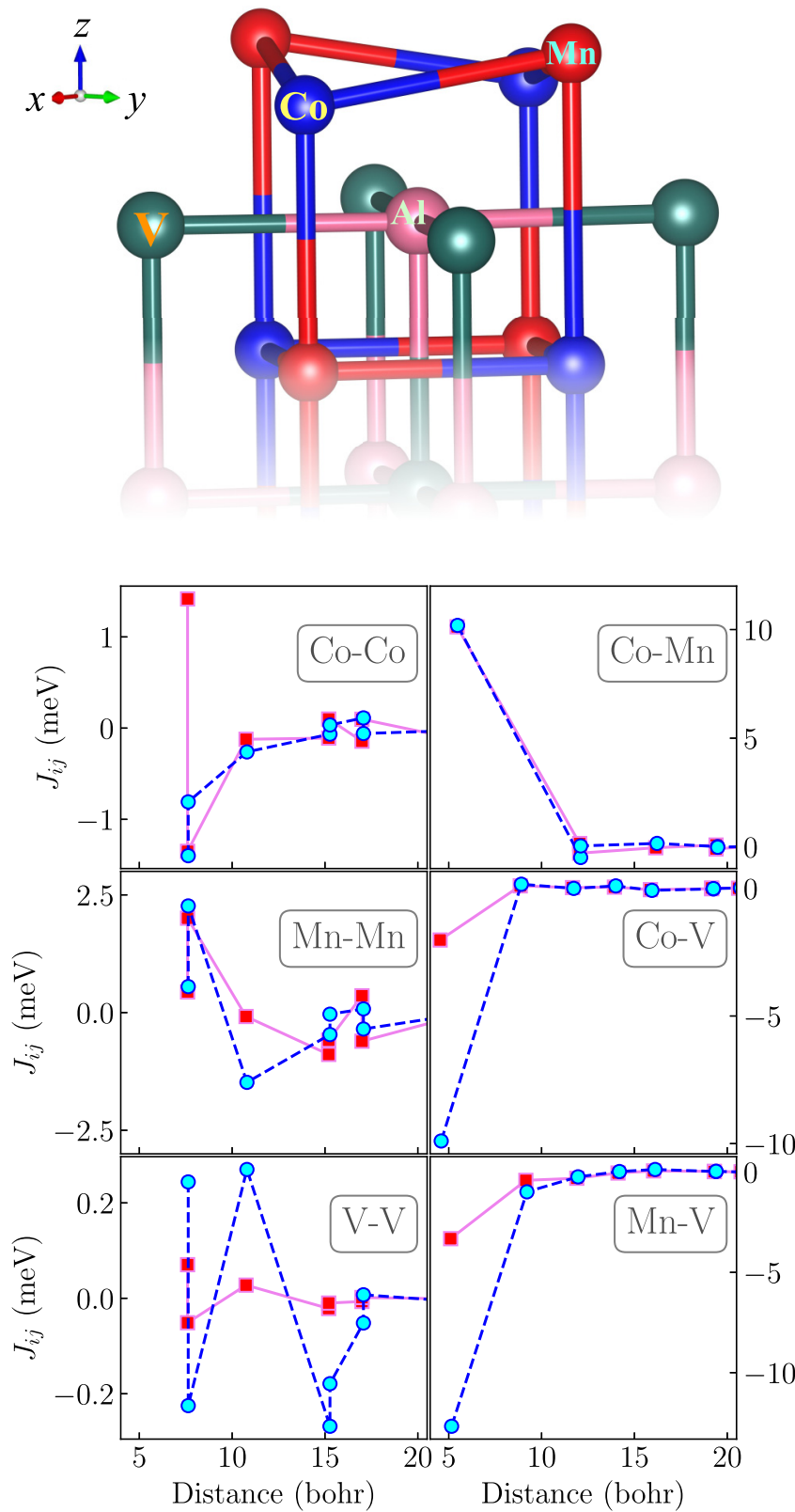

FIG. 2. Top panel: Structure of the CoMn-terminated slab of CoMnVAl. Atomic relaxations result in a visible buckling of the surface with Co atoms pushed closer to the subsurface by $\Delta z=-0.1$ $(-0.04) \AA$ and $\mathrm{Mn}$ atoms displaced in the opposite direction by $\Delta z=0.38(0.44) \AA$ in GGA $(\mathrm{GGA}+U)$. Bottom panel: Interatomic exchange parameters $J_{i j}$ 's as a function of the distance between the atoms. The results obtained with GGA $(\mathrm{GGA}+U)$ are shown with red squares (cyan circles).

predicted reference state is indeed stable. The resulting spinwave stiffness is slightly larger in GGA as compared to $\mathrm{GGA}+U$ calculations.

Conclusion and outlook. We have demonstrated that $X X^{\prime} Y Z$ Heusler materials, which are semiconducting in the bulk, host a metallic and ferrimagnetic (quasi-)2D electron liquid at their [001] $X X^{\prime}$-terminated surface. The ordering temperatures are expected to be within experimental range, as predicted for CoMnVAl. Our predictions can be verified

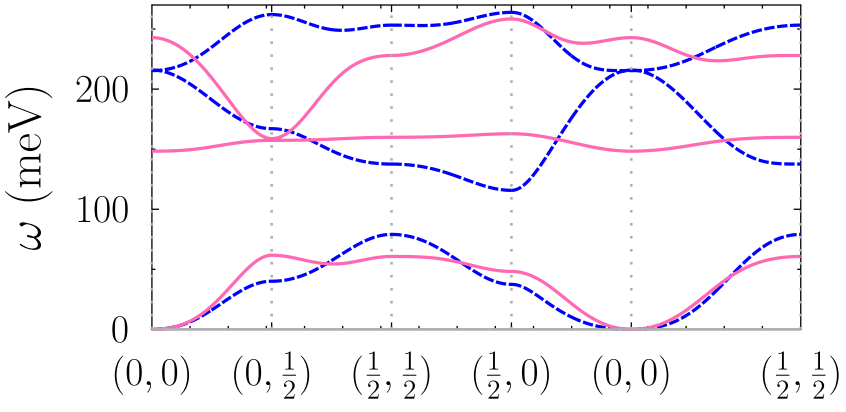

FIG. 3. Adiabatic magnon spectrum of the CoMn-terminated CoMnVAl slab along the high-symmetry lines in the plane of the surface. Solid (dashed) lines correspond to $\operatorname{GGA}(+U)$-derived data.

through several surface-sensitive experimental techniques. The magnetically ordered state can be investigated using x-ray circular dichroism $[66,67]$ or spin-polarized scanning tunneling microscopy/spectroscopy [68]. Metallicity at the surface can be verified using ARPES [69]. Finally, surface magnon states can potentially be measured with spin-polarized electron energy loss spectroscopy [70] and then compared to our predicted spectrum.

The remarkable magnetic state predicted in this Rapid Communication has a potential of being important for technological applications. Having an intrinsic metallic magnetism at the surface of a semiconducting interior implies that Heusler semiconductors may provide a practical realization of a magnetic tunnel junction within a single entity. We also note that for practical use in spintronic devices, it is the interface to a contact that matters, which should preferably be metallic and magnetic. The current materials intrinsically have these properties, thus it should not be difficult to find a suitable contact material, preserving the latter.

Our results can be strengthened by further theoretical and experimental investigations. The spin-orbit coupling has been neglected in the present analysis and its inclusion might lead to interesting features of these materials, such as nontrivial topology and Dzyaloshinskii-Moriya interactions at the surface. Finally, we speculate that the dynamical correlation effects, which are relevant for HM Heuslers [30], might be even more interesting for the surface states discussed here.

Acknowledgments. The work of I.D.M. is supported by the appointment to the JRG program at the APCTP through the Science and Technology Promotion Fund and Lottery Fund of the Korean Government, as well as by the Korean Local Governments, Gyeongsangbuk-do Province and Pohang City. L.C. gratefully acknowledges the financial support provided by the Augsburg Center for Innovative Technologies, and by the Deutsche Forschungsgemeinschaft (DFG, German Research Foundation) - Projektnummer 107745057 TRR 80/F6. O.E. acknowledges support from the Swedish Research Agency, the Knut and Alice Wallenberg Foundation, the Foundation for Strategic Research, the Swedish Energy Agency, and eSSENCE. The computer simulations were performed on computational resources provided by NSC allocated by the Swedish National Infrastructure for Computing (SNIC). 
[1] I. Tamm, Phys. Z. Sowjetunion 1, 733 (1932).

[2] W. Shockley, Phys. Rev. 56, 317 (1939).

[3] F. J. Himpsel and D. E. Eastman, Phys. Rev. Lett. 41, 507 (1978).

[4] P. O. Gartland and B. J. Slagsvold, Phys. Rev. B 12, 4047 (1975).

[5] S.-L. Weng, E. W. Plummer, and T. Gustafsson, Phys. Rev. B 18, 1718 (1978).

[6] J. A. Stroscio, D. T. Pierce, A. Davies, R. J. Celotta, and M. Weinert, Phys. Rev. Lett. 75, 2960 (1995).

[7] A. F. Santander-Syro, O. Copie, T. Kondo, F. Fortuna, S. Pailhès, R. Weht, X. G. Qiu, F. Bertran, A. Nicolaou, A. Taleb-Ibrahimi, P. Le Fèvre, G. Herranz, M. Bibes, N. Reyren, Y. Apertet, P. Lecoeur, A. Barthélémy, and M. J. Rozenberg, Nature (London) 469, 189 (2011).

[8] C. L. Kane and E. J. Mele, Phys. Rev. Lett. 95, 146802 (2005).

[9] A. Ohtomo, D. A. Muller, J. L. Grazul, and H. Y. Hwang, Nature (London) 419, 378 (2002).

[10] A. Ohtomo and H. Y. Hwang, Nature (London) 427, 423 (2004).

[11] C. H. Ahn, J. M. Triscone, and J. Mannhart, Nature (London) 424, 1015 (2003).

[12] N. Pavlenko, T. Kopp, E. Y. Tsymbal, G. A. Sawatzky, and J. Mannhart, Phys. Rev. B 85, 020407(R) (2012).

[13] N. Pavlenko, T. Kopp, E. Y. Tsymbal, J. Mannhart, and G. A. Sawatzky, Phys. Rev. B 86, 064431 (2012).

[14] R. Pentcheva and W. E. Pickett, Phys. Rev. B 74, 035112 (2006).

[15] K. Janicka, J. P. Velev, and E. Y. Tsymbal, J. Appl. Phys. 103, 07B508 (2008).

[16] T. Graf, C. Felser, and S. S. Parkin, Prog. Solid State Chem. 39, 1 (2011).

[17] I. Galanakis, P. Mavropoulos, and P. H. Dederichs, J. Phys. D: Appl. Phys. 39, 765 (2006).

[18] S. Chadov, T. Graf, K. Chadova, X. Dai, F. Casper, G. H. Fecher, and C. Felser, Phys. Rev. Lett. 107, 047202 (2011).

[19] R. Fetzer, B. Stadtmüller, Y. Ohdaira, H. Naganuma, M. Oogane, Y. Ando, T. Taira, T. Uemura, M. Yamamoto, M. Aeschlimann et al., Sci. Rep. 5, 8537 (2015).

[20] I. Di Marco, A. Held, S. Keshavarz, Y. O. Kvashnin, and L. Chioncel, Phys. Rev. B 97, 035105 (2018).

[21] D. Xiao, Y. Yao, W. Feng, J. Wen, W. Zhu, X.-Q. Chen, G. M. Stocks, and Z. Zhang, Phys. Rev. Lett. 105, 096404 (2010).

[22] L. Basit, G. H. Fecher, S. Chadov, B. Balke, and C. Felser, Eur. J. Inorg. Chem. 2011, 3950 (2011).

[23] B.-C. Min, K. Motohashi, C. Lodder, and R. Jansen, Nat. Mater. 5, 817 (2006).

[24] R. Jansen, Nat. Phys. 3, 521 (2007).

[25] I. Appelbaum, B. Huang, and D. J. Monsma, Nature (London) 447, 295 (2007)

[26] B. T. Jonker, G. Kioseoglou, A. T. Hanbicki, C. H. Li, and P. E. Thompson, Nat. Phys. 3, 542 (2007).

[27] K. Hamaya, H. Itoh, O. Nakatsuka, K. Ueda, K. Yamamoto, M. Itakura, T. Taniyama, T. Ono, and M. Miyao, Phys. Rev. Lett. 102, 137204 (2009).

[28] D. M. Edwards and J. A. Hertz, J. Phys. F: Met. Phys. 3, 2191 (1973).

[29] V. Y. Irkhin and M. I. Katsnelson, J. Phys.: Condens. Matter 2, 7151 (1990)

[30] M. I. Katsnelson, V. Y. Irkhin, L. Chioncel, A. I. Lichtenstein, and R. A. de Groot, Rev. Mod. Phys. 80, 315 (2008).
[31] L. Chioncel, Y. Sakuraba, E. Arrigoni, M. I. Katsnelson, M. Oogane, Y. Ando, T. Miyazaki, E. Burzo, and A. I. Lichtenstein, Phys. Rev. Lett. 100, 086402 (2008).

[32] RSPT - relativistic spin-polarized toolkit, http://fplmto-rspt.org/.

[33] J. M. Wills, M. Alouani, P. Andersson, A. Delin, O. Eriksson, and O. Grechnyev, Full-Potential Electronic Structure Method, Springer Series in Solid-State Sciences Vol. 167 (Springer, Berlin, 2010)

[34] J. P. Perdew, K. Burke, and M. Ernzerhof, Phys. Rev. Lett. 77, 3865 (1996).

[35] The sequence of the atoms along the diagonal is $X-Y-X^{\prime}-Z$. Note that in this case the correct notation for the structure is $\left(X X^{\prime}\right) Y Z$. In the rest of the Rapid Communication, the parentheses are dropped.

[36] We have considered both symmetric and asymmetric slabs. The former one consists of 17 layers, which prevents the formation of an electric dipole moment. On the other hand, it does not have an integer number of electrons per atom, which may lead to a spurious metallicity. To make sure that the metallicity is intrinsic and not an artifact of a finite-size model, additional calculations were made for an asymmetric 16-layer slab of CoMnVAl. Nearly identical densities of states were obtained, which is strong evidence for the emergence of genuine metallic surface states.

[37] G. Kresse and D. Joubert, Phys. Rev. B 59, 1758 (1999).

[38] G. Kresse and J. Furthmüller, Comput. Mater. Sci. 6, 15 (1996).

[39] A. Togo and I. Tanaka, Scr. Mater. 108, 1 (2015).

[40] K. Reuter and M. Scheffler, Phys. Rev. B 65, 035406 (2001).

[41] S. J. Hashemifar, P. Kratzer, and M. Scheffler, Phys. Rev. Lett. 94, 096402 (2005)

[42] B. Wu, H. Yuan, A. Kuang, H. Chen, and Y. Feng, Appl. Surf. Sci. 258, 4945 (2012).

[43] H. Han, G. Y. Gao, and K. L. Yao, J. Appl. Phys. 111, 093730 (2012).

[44] S. Khosravizadeh, S. J. Hashemifar, and H. Akbarzadeh, Phys. Rev. B 79, 235203 (2009).

[45] E. A. Zimmer and Hirschfeld, Dtsch. Z. Chir. 40, 433 (1895).

[46] J. Ruan, C. Wang, S. Yang, R. Kainuma, and X. Liu, J. Alloys Compd. 615, 998 (2014).

[47] See Supplemental Material at http://link.aps.org/supplemental/ 10.1103/PhysRevMaterials.4.021401 for a detailed analysis of the energetic and dynamical structural stability of the CoMnVAl surface, as well as for the layer-resolved density of states of the CoMn termination.

[48] V. I. Anisimov, F. Aryasetiawan, and A. I. Lichtenstein, J. Phys.: Condens. Matter 9, 767 (1997).

[49] O. Grånäs, I. Di Marco, P. Thunström, L. Nordström, O. Eriksson, T. Björkman, and J. M. Wills, Comput. Mater. Sci. 55, 295 (2012).

[50] E. Şaşığlu, I. Galanakis, C. Friedrich, and S. Blügel, Phys. Rev. B 88, 134402 (2013).

[51] F. Aryasetiawan, M. Imada, A. Georges, G. Kotliar, S. Biermann, and A. I. Lichtenstein, Phys. Rev. B 70, 195104 (2004).

[52] A. I. Liechtenstein, M. I. Katsnelson, V. P. Antropov, and V. A. Gubanov, J. Magn. Magn. Mater. 67, 65 (1987).

[53] M. I. Katsnelson and A. I. Lichtenstein, Phys. Rev. B 61, 8906 (2000). 
[54] Y. O. Kvashnin, O. Grånäs, I. Di Marco, M. I. Katsnelson, A. I. Lichtenstein, and O. Eriksson, Phys. Rev. B 91, 125133 (2015).

[55] B. Skubic, J. Hellsvik, L. Nordström, and O. Eriksson, J. Phys.: Condens. Matter 20, 315203 (2008).

[56] O. Eriksson, A. Bergman, L. Bergqvist, and J. Hellsvik, Atomistic Spin Dynamics: Foundations and Applications (Oxford University Press, Oxford, UK, 2017).

[57] J. C. Slater, Phys. Rev. 49, 931 (1936).

[58] L. Pauling, Phys. Rev. 54, 899 (1938).

[59] K. Özdoğan, E. Şaşiğlu, and I. Galanakis, J. Appl. Phys. 113, 193903 (2013).

[60] H. van Leuken and R. A. de Groot, Phys. Rev. Lett. 74, 1171 (1995).

[61] M. Tas, E. Şaşıŏlu, I. Galanakis, C. Friedrich, and S. Blügel, Phys. Rev. B 93, 195155 (2016).

[62] S. Wurmehl, G. H. Fecher, H. C. Kandpal, V. Ksenofontov, C. Felser, H.-J. Lin, and J. Morais, Phys. Rev. B 72, 184434 (2005).
[63] H. C. Kandpal, G. H. Fecher, C. Felser, and G. Schönhense, Phys. Rev. B 73, 094422 (2006).

[64] I. Galanakis, J. Phys.: Condens. Matter 14, 6329 (2002).

[65] The interactions between other atoms were not computed, since the magnetic moments are too small and of induced nature, which makes the application of the magnetic force theorem doubtful.

[66] P. Carra, B. T. Thole, M. Altarelli, and X. Wang, Phys. Rev. Lett. 70, 694 (1993).

[67] C. T. Chen, Y. U. Idzerda, H.-J. Lin, N. V. Smith, G. Meigs, E. Chaban, G. H. Ho, E. Pellegrin, and F. Sette, Phys. Rev. Lett. 75, 152 (1995).

[68] R. Wiesendanger, Rev. Mod. Phys. 81, 1495 (2009).

[69] V. Strocov, J. Electron Spectrosc. Relat. Phenom. 229, 100 (2018).

[70] J. Prokop, W. X. Tang, Y. Zhang, I. Tudosa, T. R. F. Peixoto, K. Zakeri, and J. Kirschner, Phys. Rev. Lett. 102, 177206 (2009). 